Casos Clínicos

\title{
Adenocarcinoma gástrico primario concomitante con tumor estromal gastrointestinal: Reporte de un caso y revisión de literatura
}

\author{
Lilia Antonio P, Pablo G uzmán G, Miguel Villaseca H, \\ Juan Araya O, G onzalo De Toro C, Juan Roa S. \\ Concomitant presence of a gastric \\ adenocarcinoma and gastrointestinal \\ stromal tumor. Report of one case
}

The concomitant presence of a primary gastric adenocarcinoma and a gastrointestinal stromal tumor in the stomach is uncommon. We report a 68-year-old male with an advanced gastric adenocarcinoma. During gastrectomy, a nodular intramural lesion was found. The pathological study, revealed a gastrointestinal stromal tumor, positive form CD117. After six months of follow up, there is no evidence of recurrence of either tumor (Rev Méd Chile 2009: 137: 531-6).

(Key words: Adenocarcinoma; Neoplasms, multiple primary; Stomach neoplasms)

Recibido el 28 de julio, 2008. Aceptado el 21 de agosto, 2008.

Departamento de Anatomía Patológica. Facultad de Medicina. Universidad de La Frontera. Temuco, Chile.

$E^{1}$ cáncer gástrico ocupa, a nivel mundial, el cuarto lugar en frecuencia y el segundo en mortalidad $^{1}$. En Chile es muy frecuente, siendo la principal causa de muerte por cáncer, con una tasa de mortalidad de 20 por 100.000 individuos, una de las más altas del mundo ${ }^{2}$. La gran mayoría de estas neoplasias malignas corresponden a adenocarcinomas; otros tipos histológicos como linfomas y sarcomas son mucho menos frecuentes, resultando más raro aún, el desarrollo sincrónico de un adenocarcinoma con un tumor

Correspondencia a: Dra. Lilia Isabel Antonio Padilla. Departamento de Anatomía Patológica. Facultad de Medicina, Universidad de La Frontera. Temuco, Chile. Tel/Fax: 045296591. E mail: lilia.antonio@gmail.com estromal gastrointestinal (GIST), de los cuales existen escasos reportes internacionales.

Presentamos el primer caso registrado en Chile de adenocarcinoma y GIST gástrico concomitante, que corresponde a un paciente de 68 años de edad, intervenido por adenocarcinoma gástrico corporal avanzado, cuyo estudio anatomopatológico reveló además la presencia de un GIST intramural, confirmado con técnicas de inmunohistoquímica. Adicionalmente se revisa la literatura internacional disponible de esta infrecuente asociación.

\section{CASO CLÍNICO}

Paciente de sexo masculino, de 68 años, con antecedentes de diabetes mellitus tipo 2, insulino 
requiriente, hipertensión arterial desde hace 15 años y síndrome ulceroso en tratamiento médico regular, sin historia familiar de neoplasias. Consultó por cuadro de 2 meses de evolución de intolerancia alimentaria, vómitos postprandiales progresivos y baja de peso de 8 kilos.

La ecografía abdominal describió sólo microlitiasis vesicular. En endoscopia digestiva alta se observó gran formación cardial, nodular, ulcerada, de aspecto neoplásico, de bordes mamelonados y zona central necrótica con restos fibrinohemáticos. La biopsia concluyó adenocarcinoma poco diferenciado con células en anillo de sello, infiltrante en mucosa gástrica. No se encontró Helicobacter pylori. En tomografía axial computada de abdomen, se describió a nivel de tercio superior gástrico, un engrosamiento parietal irregular, con aumento de la densidad del tejido adiposo cardial y presencia de linfonodos regionales, de entre 0,6 a 1,2 cm. Se efectuó gastrectomía total, encontrándose en el intraoperatorio una extensa lesión neoplásica subcardial en curvatura menor y una segunda lesión, nodular, intramural, en cara anterior de cuerpo gástrico. La evolución postoperatoria fue satisfactoria, sin evidencias de recurrencias después de 6 meses de seguimiento.

\section{MATERIALES Y MÉTODO}

La pieza quirúrgica fue procesada según el protocolo japonés para cáncer gástrico ${ }^{3}$, fue abierta por curvatura mayor y se disecaron los grupos ganglionares perigástricos. El estómago fue estirado en plancha de parafina y fijado en formalina neutra tamponada a 10\%. Las muestras representativas obtenidas se incluyeron en parafina para el procesamiento histológico y fueron teñidas con hematoxilina-eosina (HE), ácido periódico de Schiff y azul alcian. Para análisis inmunohistoquímico se seleccionaron y cortaron inclusiones de tejido en parafina. Se realizó la técnica de complejo avidin-biotina con los anticuerpos CD34 (Tipo II, QBEnd/10, NeoMarkers) y CD117 (c-Kit, Policlonal, NeoMarkers). Como cromógeno se utilizó diamenobenzidina (DAB). Los núcleos se contrastaron con hematoxilina.

Para la revisión de la literatura se efectuó búsqueda y selección de artículos bibliográficos, en inglés y español, desde MEDLINE usando términos libres y MESH, entre otros: gastrointestinal stromal tumor, neoplasm, stomach neoplasms. Un número extra de publicaciones se obtuvo de las referencias de los artículos previamente seleccionados, también mediante la función "related papers" en MEDLINE y búsquedas dirigidas en tablas de contenido de revistas especializadas de patología, oncología, gastroenterología y cirugía. La selección bibliográfica sólo incluyó artículos en inglés o español, con texto en extenso o resumen completo.

\section{Resultados}

El examen macroscópico de la pieza quirúrgica presenta dos lesiones. Una de ellas, en superficie mucosa, hacia curvatura menor, en unión de tercio superior con tercio medio, ulcerada, de bordes irregulares, compresivos e infiltrantes y lecho indurado, tipo Borrmann III, de 6,5 por 3,8 $\mathrm{cm}$, alejada de bordes quirúrgicos (Figura $1 \mathrm{~A}$ ). $\mathrm{Al}$ corte, presenta compromiso transmural, extendiéndose a tejido adiposo perigástrico y serosa (Figura 2A). La segunda lesión, es corporal, anterior, nodular, intramural, de 1,8 cm, que solevanta mucosa y serosa, ambas indemnes (Figura 1B). Al corte, es bien delimitada, pardo grisácea, de aspecto fascicular y consistencia elástica (Figura 2B). No existe continuidad macroscópica entre ambas lesiones. Resto de la mucosa con zonas de metaplasia intestinal.

Microscópicamente, se observa pared gástrica de arquitectura distorsionada, por proliferación epitelial maligna transmural, compuesta por células redondeadas de núcleos atípicos, hipercromáticos, en partes conformando túbulos mal definidos. Algunas células poseen vacuolas de tamaño mediano y grande, positivas para mucosustancias, que desplazan el núcleo hacia la periferia (Figura 3A). Las células neoplásicas infiltran la pared de manera difusa, comprometiendo hasta la serosa. Se encontraron metástasis de adenocarcinoma en 3 de 31 ganglios linfáticos. El segundo tumor estaba compuesto por una proliferación fusocelular monomorfa, bien delimitada, arremolinada, con 3 mitosis en 50 campos de aumento mayor, sin atipias ni áreas necróticas (Figura 3B). El tejido entre las dos lesiones presentaba sólo gastritis crónica, inespecífica. No 


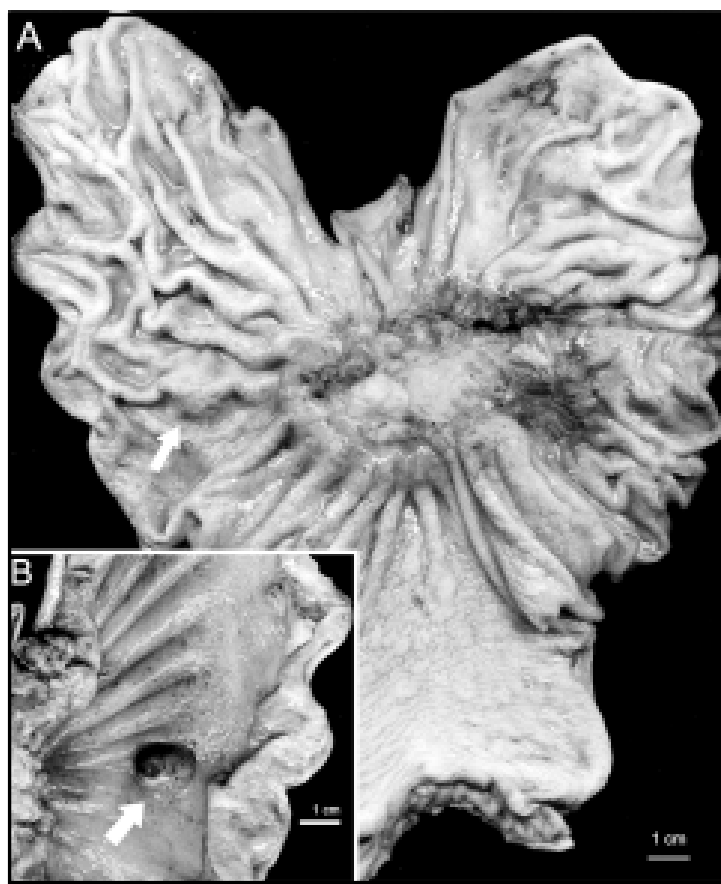

Figura 1. Pieza de gastrectomía total, vista por superficie mucosa (A), con adenocarcinoma ulcerado corporal, y zona solevantada por GIST $(\uparrow)$. En recuadro (B) cara serosa anterior gástrica, con GIST nodular $(\uparrow)$.

se encontró Helicobacter pylori. El estudio inmunohistoquímico de la segunda neoplasia presentó extensa positividad celular citoplasmática para ckit (CD117) (Figura 3C) y CD34, lo cual confirmó el diagnóstico de GIST, el cual es de naturaleza benigna, según los hallazgos morfológicos.

\section{DisCUSIÓN}

En la literatura se han descrito muy pocos casos de desarrollo concomitante de adenocarcinoma gástrico y GIST. La serie más numerosa reportada es de 5 casos $^{4}$, los restantes son descripciones esporádicas $^{5-13}$ un tumor de colisión ${ }^{14}$ y 2 casos de adenocarcinoma, GIST y una tercera neoplasia gastrointestinal $^{15,16}$.

Los casos recopilados de la búsqueda bibliográfica (Tabla 1), muestran una edad promedio de presentación de 74 años con rango de 64 a 82 años, con mayor frecuencia en sexo masculino (60\%). Los adenocarcinomas eran predominante-

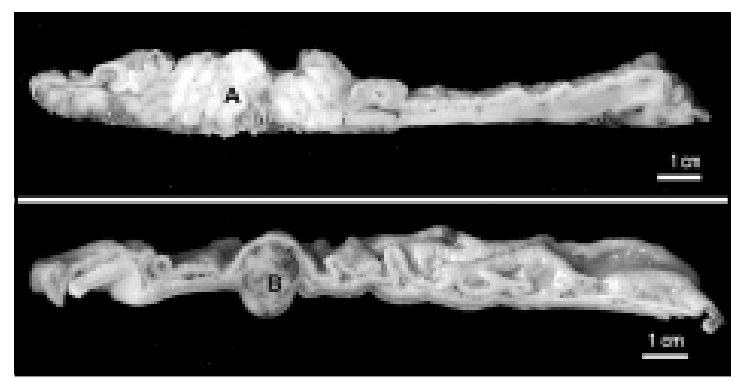

Figura 2. Superficie de corte de pared gástrica con adenocarcinoma avanzado (A) y GIST intramural (B).

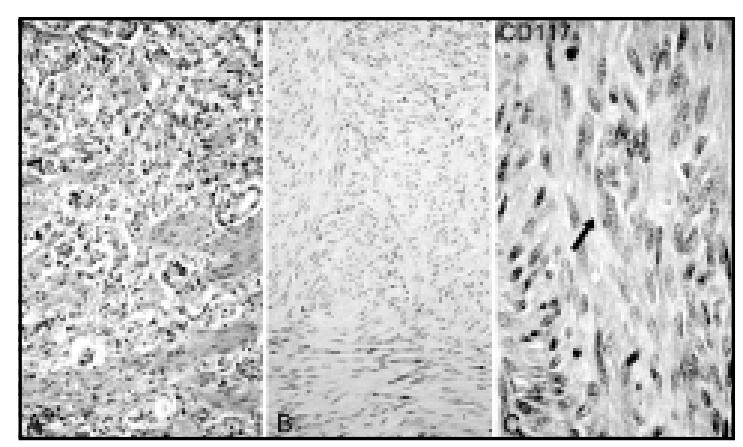

Figura 3. Microfotografía de adenocarcinoma gástrico tubular poco diferenciado (A) (H\&E, 100x). Tumor estromal gástrico de patrón fusocelular, sin pleomorfismo o necrosis (H\&E, 100x) (B) y con inmunopositividad de las células fusadas para CD117 ( $\uparrow$ ) (C) (DAB, 400x).

mente lesiones avanzadas (65\%), de 5,3 cm de tamaño promedio, en general ulcerados, antrales, en $65 \%$ de tipo intestinal de Lauren y con metástasis ganglionares linfáticas en 8 de 13 casos. Los adenocarcinomas incipientes midieron en promedio $1,6 \mathrm{~cm}$, principalmente antrales, sin predominio de una morfología característica ni evidencias de metástasis ganglionares linfáticas. Ambos tipos de lesiones no presentaron marcadas diferencias en sexo y edad de presentación. Los GIST correspondieron a lesiones nodulares, de 2 $\mathrm{cm}$ en promedio, bien delimitados, de predominio subseroso, ubicados principalmente en cuerpo gástrico (60\%), de aspecto histológico fusocelular y comportamiento benigno. Respecto a la localización de las neoplasias, en 6 casos de adenocarcinoma antral, el GIST se ubicó en cuerpo gástrico y en 8 casos, ambas neoplasias se presentaron en el mismo tercio gástrico. Sólo en 1 caso, el diagnósti- 
Tabla 1. Resumen de casos de adenocarcinoma gástrico concomitante con tumor estromal gastrointestinal descritos en la literatura

\begin{tabular}{|c|c|c|c|c|c|c|}
\hline $\begin{array}{l}\text { Caso } \\
\text { Sexo }\end{array}$ & Edad & M acroscopia & Microscopia & Estadio & O bservaciones & Ref. \\
\hline 1 & $81 / \mathrm{M}$ & $\begin{array}{l}\text { A: Masa vegetante, cardial de } 4 \mathrm{~cm} \\
\text { E: Nódulo intramural, fúndico de } 5 \mathrm{~cm}\end{array}$ & $\begin{array}{l}\text { Tipo intestinal } \\
\text { Epiteloideo, borderline }\end{array}$ & T2bNOM0 & & 4 \\
\hline 2 & 79/M & $\begin{array}{l}\text { A: Erosión antral de } 2 \mathrm{~cm} \\
\text { E: Nódulo submucoso pilórico de } 6 \mathrm{~cm}\end{array}$ & $\begin{array}{l}\text { Tipo difuso } \\
\text { Fusocelular, maligno }\end{array}$ & T1bNOM0 & & 4 \\
\hline 3 & $75 / \mathrm{H}$ & $\begin{array}{l}\text { A: Úlcera antral de } 4 \mathrm{~cm} \\
\text { E: Nódulo submucoso antral de } 5 \mathrm{~cm}\end{array}$ & $\begin{array}{l}\text { Tipo intestinal } \\
\text { Fusocelular borderline }\end{array}$ & T2aN1M0 & & 4 \\
\hline 4 & $79 / \mathrm{M}$ & $\begin{array}{l}\text { A: Úlcera pilórica de } 1,2 \mathrm{~cm} \\
\text { E: Nódulo subseroso corporal de } 5 \mathrm{~cm}\end{array}$ & $\begin{array}{l}\text { Tipo intestinal } \\
\text { Fusocelular borderline }\end{array}$ & T2aN1M0 & & 4 \\
\hline 5 & $79 / \mathrm{H}$ & $\begin{array}{l}\text { A: Úlcera antral de } 2 \mathrm{~cm} \\
\text { E: Nódulo subseroso corporal de } 0,6 \mathrm{~cm}\end{array}$ & $\begin{array}{l}\text { Tipo intestinal } \\
\text { Fusocelular benigno }\end{array}$ & T2aNOMO & & 4 \\
\hline 6 & $71 / \mathrm{H}$ & $\begin{array}{l}\text { A: Tumor vegetante, ulcerado, antral de } 5,7 \mathrm{~cm} \\
\text { E: Nódulo subseroso corporal de } 0,5 \mathrm{~cm}\end{array}$ & $\begin{array}{l}\text { Tipo difuso } \\
\text { Fusocelular benigno }\end{array}$ & T3N+M0 & & 5 \\
\hline 7 & $77 / \mathrm{H}$ & $\begin{array}{l}\text { A: Masa vegetante, corporal de } 7,5 \mathrm{~cm} \\
\text { E: Nódulo submucoso cardial de } 0,6 \mathrm{~cm}\end{array}$ & $\begin{array}{l}\text { Tipo intestinal } \\
\text { Fusocelular benigno }\end{array}$ & T2N1M0 & & 5 \\
\hline 8 & $70 / \mathrm{M}$ & $\begin{array}{l}\text { A: Erosión angular de } 1,7 \mathrm{~cm} \\
\text { E: Pólipo sésil fúndico de } 1,1 \mathrm{~cm}\end{array}$ & $\begin{array}{l}\text { Tipo difuso } \\
\text { Fusocelular benigno }\end{array}$ & T1N0M0 & & 6 \\
\hline 9 & $70 / \mathrm{M}$ & $\begin{array}{l}\text { A: Engrosamiento parietal corporal-antral de } 10 \mathrm{~cm} \\
\text { E: Nódulo submucoso de } 2 \mathrm{~cm}\end{array}$ & $\begin{array}{l}\text { Tipo difuso } \\
\text { Fusocelular benigno }\end{array}$ & T3N1M0 & & 7 \\
\hline 10 & $77 / \mathrm{M}$ & $\begin{array}{l}\text { A: Úlcera antral } \\
\text { E: Nódulo subseroso antral de } 1 \mathrm{~cm}\end{array}$ & $\begin{array}{l}\text { Tipo intestinal } \\
\text { Fusocelular benigno }\end{array}$ & T1N0M0 & & 8 \\
\hline 11 & $64 / \mathrm{M}$ & $\begin{array}{l}\text { A: Tumor antral de } 5 \mathrm{~cm} \\
\text { E: Nódulo subseroso corporal de } 2 \mathrm{~cm}\end{array}$ & $\begin{array}{l}\text { Tipo difuso } \\
\text { Fusocelular benigno }\end{array}$ & T4N0M0 & & 9 \\
\hline 12 & $66 / \mathrm{H}$ & $\begin{array}{l}\text { A: Tumor angular de } 1 \mathrm{~cm} \\
\text { E: Nódulo intramural corporal de } 1 \mathrm{~cm}\end{array}$ & $\begin{array}{l}\text { Tipo intestinal } \\
\text { Fusocelular benigno }\end{array}$ & T1N0M0 & & 9 \\
\hline 13 & $74 / \mathrm{H}$ & A: Lesión elevada superficial, antral de $1,5 \mathrm{~cm}$ & Tipo mixto & T1N0M0 & $\begin{array}{l}\text { Hepatitis } \\
\text { autoinmune }\end{array}$ & 10 \\
\hline 14 & $82 / \mathrm{H}$ & $\begin{array}{l}\text { E: } 2 \text { nódulos serosos, corporales de } 0,8 \text { y } 1 \mathrm{~cm} \\
\text { A: Masa transmural corporal de } 9,5 \mathrm{~cm} \\
\text { E: Nódulo mucoso ulcerado corporal de } 1,5 \mathrm{~cm}\end{array}$ & $\begin{array}{l}\text { Fusocelular benigno } \\
\text { Tipo difuso } \\
\text { Fusocelular maligno }\end{array}$ & T4N0M0 & & 11 \\
\hline 15 & $78 / \mathrm{H}$ & A: Úlcera mucosa antral & Tipo intestinal & T1N0M0 & $\begin{array}{l}\text { Púrpura } \\
\text { trombocito- } \\
\text { pénico } \\
\text { idiopático }\end{array}$ & 12 \\
\hline 16 & 78/M & $\begin{array}{l}\text { E: Nódulo subseroso antral de } 0,9 \mathrm{~cm} \\
\text { A: Masa ulcerada antral de } 6 \mathrm{~cm} \\
\text { E: Nódulo antral de } 1 \mathrm{~cm}\end{array}$ & $\begin{array}{l}\text { Fusocelular benigno } \\
\text { Tipo intestinal } \\
\text { Fusocelular maligno }\end{array}$ & T3N1M0 & & 13 \\
\hline 17 & $70 / \mathrm{H}$ & $\begin{array}{l}\text { AE: Tumor elevado, ulcerado e infiltrante corporal, } \\
\text { de } 8 \mathrm{~cm}\end{array}$ & $\begin{array}{l}\text { Tipo intestinal } \\
\text { Fusocelular benigno }\end{array}$ & T3N1M0 & $\begin{array}{l}\text { Tumor de } \\
\text { colisión }\end{array}$ & 14 \\
\hline 18 & $72 / \mathrm{H}$ & A: Úlcera antral de $1,5 \mathrm{~cm}$ & Tipo intestinal & T2aNOM0 & $\begin{array}{l}\text { Adenocar- } \\
\text { cinoma } \\
\text { vesicular }\end{array}$ & 15 \\
\hline 19 & $78 / \mathrm{H}$ & $\begin{array}{l}\text { E: Nódulo subseroso corporal de } 2,5 \mathrm{~cm} \\
\text { A: Solevantamiento leve antral } \\
\text { E: Nódulo seroso corporal de } 1,5 \mathrm{~cm}\end{array}$ & $\begin{array}{l}\text { Fusocelular benigno } \\
\text { Tipo mixto } \\
\text { Fusocelular benigno }\end{array}$ & T1N0M0 & Linfoma MALT & 16 \\
\hline 20 & $68 / \mathrm{H}$ & $\begin{array}{l}\text { A: Úlcera corporal de } 6,5 \mathrm{~cm} \\
\text { E: Nódulo intramural corporal de } 1,8 \mathrm{~cm}\end{array}$ & $\begin{array}{l}\text { Tipo mixto } \\
\text { Fusocelular benigno }\end{array}$ & T3N1M0 & & $\begin{array}{r}\text { Caso } \\
\text { actual }\end{array}$ \\
\hline
\end{tabular}

(A: Adenocarcinoma, E: GIST). 
co de GIST sincrónico, se conocía en el periodo preoperatorio ${ }^{6}$, la mayoría fueron hallazgos intraoperatorios o durante el examen macroscópico.

El GIST es la neoplasia gástrica mesenquimática más frecuente, se originarían de las células intersticiales de Cajal o sus precursoras, que cumplen un rol regulador de la motilidad gastrointestinal. En más de $80 \%$ de los casos se ha detectado mutación de genes receptores de tirosin-quinasa, como el c-kit o PDGFRA (platelet-derived growth factor receptor alpha). La intensa positividad de la proteína KIT (CD117) en las células de Cajal, presente también en más del 95\% de los GIST, se ha transformado en la herramienta más sensible y específica para su diagnóstico, permitiendo diferenciarlo de otros tumores mesenquimáticos ${ }^{17}$. En nuestra revisión, todos los casos recientes, presentaban positividad para CD117, aquellos casos anteriores al año 2002, resultaron en su mayoría positivos para CD34, que fue el primer marcador relativamente específico para GIST y se expresa en un 50 a $80 \%$ de estos.

Las hipótesis respecto al desarrollo concomitante de ambos tumores apuntan a 1) hallazgo incidental, podría explicar sólo por simple coincidencia esta asociación, en especial en áreas de alto riesgo como nuestro país, 2) mutación genética que podría predisponer a la génesis sincrónica

\section{REFERENCIAS}

1. Parkin DM, Bray F, Ferlay J, Pisani P. Global cancer statistics, 2002. CA Cancer J Clin 2005; 55: 74-108.

2. MEDiNA E. Epidemiología del cáncer gástrico. En: Csendes A, editor. Cáncer Gástrico. Editorial Mediterráneo Ltda.; 2006. p. 17-26.

3. KaJITANI T. Japanese Research Society for Gastric Cancer. The general rules for the gastric cancer study in surgery and pathology. Jpn J Surg 1981; 11: 127-45.

4. Maiorana A, Fante R, Cesinaro A, Fano R. Synchronous occurrence of epithelial and stromal tumors in the stomach: a report of 6 cases. Arch Pathol Lab Med 2000; 124: 682-6.

5. Bircan S, Candir O, Aydin S, Baspinar S, Bulbul M, KapuCuoglu N, ET AL. Synchronous primary adenocarcinoma and gastrointestinal stromal tumor in the stomach: A report of two cases. Turk J Gastroenterol 2004; 15: 187-91.

6. LiN L, TZENG E, Wei K, LiN W. Small gastrointestinal stromal tumor concomitant with early gastric cancer: a case report. World J Gastroenterol 2006; 12: 812-5. de esta asociación o 3) un agente carcinogénico en común que desencadenaría en tejido epitelial y no epitelial del mismo órgano, ambas neoplasias ${ }^{4}$. Este último postulado ha encontrado evidencias experimentales, ya que al administrar N-metil- $\mathrm{N}^{\prime}$ nitro-N-nitrosoguanidina (MNNG) a ratas, se logra inducir no sólo adenocarcinomas gástricos, sino también tumores no epiteliales, cuando junto a MNNG se asocia estrés 0 ácido acetilsalicílico ${ }^{18}$. Además, recientemente, se ha logrado, mediante un modelo de inducción de reflujo duodenal, desarrollar tumores epiteliales y no epiteliales gástricos, presentando algunos de estos últimos inmunopositividad para CD117 19 . Sin embargo no existe evidencia que muestre que estas sustancias y modelos cumplan un rol fundamental en neoplasias gástricas humanas.

En conclusión, se presenta el primer caso de desarrollo concomitante de adenocarcinoma y GIST gástrico, en un país de alta incidencia y mortalidad de cáncer gástrico. Esta asociación no dispone de evidencias sólidas que apoyen una predisposición genética o una conexión causal con carcinógenos por lo que se deben estudiar una mayor cantidad de casos para explicar la aparición simultánea de neoplasias de diferente tipo histológico.

7. Rauf F, Ahmad Z, Muzzafar S, Hussaini S. Synchronous occurrence of gastrointestinal stromal tumor and gastric adenocarcinoma: a case report. J Pak Med Assoc 2006; 56: 184-6.

8. Rubiales S, Ovelar Y, Beltran De Heredia J, Del Vale L. Synchronous diagnosis of gastric adenocarcinoma and GIST. An Med Interna 2005; 22: 606-7.

9. Wronski M, ZiarkieWicZ-WroblewsKa B, Gornicka B, CeBulski W, SLodkowski M, Wasiutynski A, et al. Synchronous occurrence of gastrointestinal stromal tumors and other primary gastrointestinal neoplasms. World J Gastroenterol 2006; 12: 5360-2.

10. UChiYama S, Nagano M, TAKaHAShi $\mathrm{N}$, Hidaka $\mathrm{H}$, Matsuda H, Nagaike K et al. Synchronous adenocarcinoma and gastrointestinal stromal tumors of the stomach treated laparoscopically. Int J Clin Oncol. 2007; 12: 678-81.

11. Lee F, Jan J, Wang J, Yu C, Wu C. Synchronous gastric gastrointestinal stromal tumor and signet-ring cell adenocarcinoma: a case report. Int J Surg Pathol 2008; 15: 397-400.

12. Viluas C, Gourgiotis S, Veloudis G, Sampaziotis D, MoreAs H. Synchronous early gastric cancer and 
gastrointestinal stromal tumor in the stomach of a patient with idiopathic thrombocytopenic purpura. J Dig Dis 2008; 9: 104-7.

13. Salemis N, Gourgiotis S, Tsiambas E, Karameris A, TSOHATARIDIS E. Synchronous occurrence of advanced adenocarcinoma with a stromal tumor in the stomach: a case report. J Gastrointestin Liver Dis 2008; 17: 213-5.

14. Lu W, CHEN H, HsieH P. Collision tumor of the stomach: a case report of mixed gastrointestinal stromal tumor and adenocarcinoma. J Clin Gastroenterol 2002; 35: 332-4.

15. Chen H, Chen C, Tzeng M, Tsay H, Chiang H, Lu C, et AL. Resection of triple synchronous tumors-gastric adenocarcinoma, gallbladder adenocarcinoma and stromal tumor of the stomach. Chin Med J (Taipei) 2001; 64: 655-60.

16. Kaffes A, Hughes L, Hownshead J, Katelaris P. Synchronous primary adenocarcinoma, mucosa- associated lymphoid tissue lymphoma and gastrointestinal stromal tumor in a Helicobacter pyloriinfected stomach. J Gastroenterol Hepatol 2002; 17: 1033-6.

17. Miettinen M, Lasota J. Gastrointestinal Stromal Tumors. Review on morphology, molecular pathology, prognosis, and differential diagnosis. Arch Pathol Lab Med 2006; 130: 1466-78.

18. Cohen A, Gelier S, Horowitz I, Toth L, Werther L. Experimental models for gastric leiomyosarcoma. The effects of N-methyl-N'-nitro-N-nitrosoguanidine in combination with stress, aspirin or sodium taurocholate. Cancer 1984; 53: 1088-92.

19. Mukaisho K, Miwa K, Totsuka $Y$, Shimomura A, SUGIHARA H, WAKABAYASHI K, ET AL. Induction of gastric GIST in rat and establishment of GIST cell line. Cancer Lett 2006; 231: 295-303. 